\title{
POTENCIAL TÓXICO DE SEDIMENTOS DRAGADOS DAS BAÍAS DE SEPETIBA E DA GUANABARA (RJ) EM CENÁRIO DE DISPOSIÇÃO EM LATOSSOLO
}

\author{
POTENTIAL TOXICITY OF DREDGED SEDIMENTS FROM SEPETIBA AND GUANABARA \\ BAYS (RJ) IN SCENARIO OF DISPOSAL IN FERRALSOLS
} \author{
CAMPOS $^{6}$, Helena POLIVANOV ${ }^{7}$, Selma Gomes Ferreira LEITE \\ Cidade Universitária, Rio de Janeiro - RJ.E-mail: aline.lessa@poli.ufrj.br; selma@eq.ufrj.br \\ Centro Niterói - RJ. E-mail: tantufaz17@gmail.com; wmachado@geoq.uff.br \\ E-mail: christiane.monte@yahoo.com.br \\ Universitária, Rio de Janeiro, RJ. E-mail: zcastilhos@cetem.gov.br \\ 225/301-L, Gávea, Rio de Janeiro, RJ. E-mail: tacio@puc-rio.br \\ Universitária, Rio de Janeiro - RJ. E-mail: hpolivanov@gmail.com

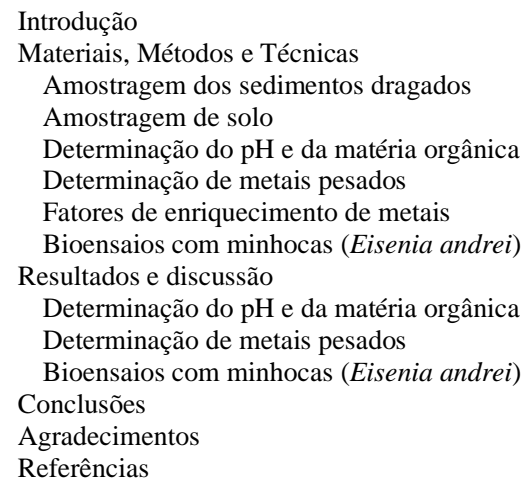

Aline Ribeiro Lessa FERREIRA ${ }^{1}$, Ricardo Gonçalves CESAR ${ }^{2}$, Danielle Martins de SIQUEIRA ${ }^{2}$, Ana Paula de Castro RODRIGUES ${ }^{3}$, Mariana VEZZONE ${ }^{2}$, Christiane MONTE $^{4}$, Wilson Thadeu MACHADO ${ }^{3}$, Zuleica Carmen CASTILHOS ${ }^{3,5}$, Tácio Mauro de

${ }^{1}$ Universidade Federal do Rio de Janeiro, UFRJ. Escola Politécnica \& Escola de Química. Avenida Athos da Silveira Ramos, 149 -

${ }^{2}$ Universidade Federal do Rio de Janeiro, UFRJ. Departamento de Geografia. Avenida Athos da Silveira Ramos, 149 - Cidade

Universitária, Rio de Janeiro - RJ. E-mail: ricardogc.geo@gmail; dani.msiq@gmail.com; mvezzone@gmail.com

${ }^{3}$ Universidade Federal Fluminense, UFF. Departamento de Geoquímica. Outeiro de São João Batista s/nº, Campus do Valonguinho,

${ }^{4}$ Universidade Federal do Oeste do Pará, UFOPA. Departamento de Geologia. Avenida Vera Paz, s/n. Salé, Santarém, PA.

${ }^{5}$ Centro de Tecnologia Mineral, CETEM/MCTI. Coordenação de Processos Minerais. Avenida Pedro Calmon, 900. Cidade

${ }^{6}$ Pontifícia Universidade Católica do Rio de Janeiro, PUC-Rio. Departamento de Engenharia Civil. Rua Marquês de São Vicente

${ }^{7}$ Universidade Federal do Rio de Janeiro, UFRJ. Departamento de Geologia. Avenida Athos da Silveira Ramos, 149 - Cidade

RESUMO - O descarte indiscriminado de efluentes domésticos e industriais tem degradado ecossistemas estuarinos do Estado do Rio de Janeiro. Ações de dragagem são comumente requeridas para recuperação destes ambientes, tornando importante a avaliação dos impactos associados à disposição terrestre de materiais dragados. O presente estudo trata da avaliação do potencial tóxico de sedimentos dragados da foz do saco do Engenho (Baía de Sepetiba, RJ), foz do Rio Meriti (Baía de Guanabara, RJ) e foz do Canal de São Francisco (Baía de Sepetiba, RJ), em cenário de disposição em Latossolo. As concentrações de metais pesados nos sedimentos foram determinadas por ICP-OES, sendo também estimados os fatores de enriquecimento (FE). As doses de sedimento aplicadas ao solo variaram entre 0 e $40 \%$, onde $0 \%$ = Latossolo puro. Bioensaios agudos com minhocas (Eisenia andrei) foram realizados nas misturas solo:sedimento conforme protocolo padrão da American Society for Testing and Materials (ASTM). A dose letal de sedimento a 50\% dos organismos (CL50), expressa em \% de sedimento adicionada ao solo, foi estimada através da análise de PriProbit. As concentrações nos sedimentos estavam acima dos valores orientadores do CONAMA 454 e 420 para disposição destes materiais em água e em solo, com exceção do sedimento do canal de São Francisco, cujos teores de metais estavam em conformidade para disposição em solo. O sedimento de maior toxicidade às minhocas foi o do Saco do Engenho (CL50 = 13,06\%), seguido da foz do Rio Meriti (CL50 = 14,60\%) e do Canal de São Francisco (CL50 = 28,02\%). Estes dados parecem concordar com as concentrações de metais determinadas para estes materiais, cujas concentrações foram maiores para o sedimento do Saco do Engenho, seguido do Rio Meriti. Além disso, os Fatores de Enriquecimento de metais logrados no Saco do Engenho e na foz do Rio Meriti indicam a ocorrência de fontes antrópicas importantes de metais pesados para estas áreas. Por fim, os sedimentos dragados foram tóxicos às minhocas, e os valores orientadores da legislação precisam ser revistos à luz das propriedades das principais ocorrências pedológicas brasileiras.

Palavras-chave: Dragagem. Metais. Minhocas. Solos. Toxicidade.

ABSTRACT - Inadequate discharges of domestic and industrial wastes have degraded estuarine ecosystems from Rio de Janeiro State (RJ). Dredging operations are often required to restore these environments and, because of that, the evaluation of potential toxicity of dredged materials in soils has become important. This work proposes the ecotoxicological evaluation of dredged sediments from the mouth of Saco do Engenho (Sepetiba bay, RJ), Meriti River (Guanabara bay, RJ) and Sao Francisco Channel (Sepetiba bay, RJ) in scenario of disposal in ferralsols. Metal concentrations in sediments were determined by ICP-OES, and metal enrichment factors (FE) 
were also estimated. The doses of sediment applied onto soils varied between 0 and $40 \%$, where $0 \%=$ pure ferralsol. Acute bioassays with earthworms (Eisenia andrei) were performed in mixtures of soil:sediment according to standard protocol (ASTM). Median lethal concentration on 50\% of the organisms (CL50), expressed in \% of sediment mixed with soil, was estimated through PriProbit analysis. Metal concentrations in the sediments were above the limits established by Brazilian law (CONAMA 454 \& 420 ) for disposal of dredged sediments in aquatic environments and soil, except for the sediment collected in the São Francisco channel, whose metal contents are in agreement for land disposal. The highest toxicity level was found for the sediment from the Saco do Engenho (CL50 = $13.06 \%$ ), followed by Meriti River (CL50 = 14.60\%) and São Francisco channel (CL50 = 28.02\%). Such data seem to support metal concentrations determined in the sediments, whose highest concentrations were found for the Saco do Engenho, followed by Meriti River. In addition, the values of metal-enrichment factors suggest the occurrence of important metal anthropogenic sources for the sediments from Saco do Engenho and Meriti River. Finally, the sediments were toxic to the earthworms and the threshold limits established by Brazilian legislation should be revised taking into consideration the properties of the main Brazilian pedological occurrences.

Keywords: Dredging. Metals. Earthworms. Soils. Toxicity.

\section{INTRODUÇÃO}

A carência de saneamento básico e o consequente lançamento de esgoto sem prétratamento nos corpos hídricos, atrelado a descartes irregulares de resíduos e efluentes industriais, vêm causando o assoreamento e contaminação de ecossistemas aquáticos costeiros do Estado do Rio de Janeiro (RJ). A maior parte destes ambientes está eutrofizada e contaminada por esgoto doméstico, hidrocarbonetos de petróleo, metais pesados, patógenos, hormônio e fármacos (Nascimento et al., 2016; Cordeiro et al., 2017; Rodrigues et al., 2017). Diante desse cenário, tornam-se indispensáveis operações de dragagem visando à manutenção das vias de transporte aquaviário, aumento da circulação das águas, viabilização segura da pesca para consumo humano, criação de áreas de lazer e, sobretudo, minimização dos efeitos deletérios ao ecossistema (Munns et al., 2002; Cesar et al., 2014).

Tendo em vista que o transporte do material dragado é frequentemente dispendioso, representando cerca de $50 \%$ a $60 \%$ dos custos totais da obra de dragagem, a eliminação desses sedimentos em áreas continentais adjacentes é comum no Brasil e pode causar sérios danos ao ecossistema (Almeida et al., 2001; Cesar et al., 2014). Contudo, a atual legislação brasileira que define as diretrizes para disposição de sedimentos dragados em solos (Resolução 420 do CONAMA/2009) e em água (Resolução 454 do CONAMA/2012) está calcada em valores orientadores para ecossistemas de clima temperado e, dessa forma, não refletem as necessidades particulares da região tropical. Uma vez que as propriedades físicas e químicas dos solos desempenham papel crucial na mobilidade e biodisponibilidade de contaminantes para a biota terrestre (Alamino et al., 2009; Van Gestel et al., 2011; Cesar et al., 2012), a revisão dos referidos valores orientadores é urgente visando sua adequação às condições tropicais.

Alguns autores têm demonstrado que a utilização de bioensaios com organismos de fauna edáfica é promissora na prevenção e prognóstico de efeitos tóxicos decorrentes da aplicação de resíduos em solo, incluindo lodo de esgoto, resíduos de mineração, sedimentos dragados e resíduos da bioprodução de etanol (Cesar et al., 2012; Bianchi, 2013; Alves et al., 2015). Cesar et al. (2014), por exemplo, apontam que o bioensaio agudo com minhocas (Eisenia andrei) é um método apropriado à avaliação do potencial tóxico oriundo da deposição terrestre de sedimentos dragados do Canal do Cunha (Baía de Guanabara, RJ). O uso de minhocas como bioindicadores decorre da sua abundância em solos tropicais e temperados, ingerem grande quantidade de solo e são sensíveis à presença de agentes tóxicos no solo (Neuhauser et al., 1985; Hinto \& Veiga, 2002).

Este trabalho trata da avaliação do potencial tóxico de sedimentos dragados oriundos de sistemas estuarinos na Baía de Guanabara e Baía de Sepetiba (RJ) em cenário de disposição em solo tropical (Latossolo). As principais hipóteses de trabalho são: (i) existe risco ecológico à saúde da fauna edáfica associado à disposição terrestre de materiais dragados; (ii) as propriedades do solo exercem papel de fundamental importância na toxicidade de sedimentos de dragagem; e (iii) os valores de metais definidos pela legislação brasileira para disposição terrestre de sedimentos dragados não são adequados para prevenir a ocorrência de efeitos nocivos sobre à biota do solo.

\section{MATERIAIS, MÉTODOS E TÉCNICAS}

\section{Amostragem dos sedimentos dragados}

Três (3) amostras de sedimento dragado foram obtidas em três (3) distintas estações de coleta (uma amostra de sedimento por estação), em setembro de
2012. A localização das estações de coleta está demonstrada na figura 1. Duas (2) estações de coleta estão localizadas na Baía de Sepetiba, na foz do Saco de Engenho e foz do Canal de São 
Francisco, e uma (1) na Baía de Guanabara, na Foz do Rio Meriti. Para cada estação de coleta, foram coletadas dez (10) amostras de sedimento superficiais (10 $\mathrm{cm}$ de profundidade), as quais foram misturadas visando obter uma amostra composta espacialmente representativa da estação. Em campo, os sedimentos foram acondicionados em sacos plásticos. No laboratório, antes serem misturados, os sedimentos foram secos à temperatura ambiente e moídos em moinho de barra.

A bacia do Rio Meriti (Baía de Guanabara, RJ) drena a região da Baixada Fluminense e recebe altas cargas de resíduos domésticos e industriais contendo metais pesados (Machado et al., 2008; Monte, 2014). Além disso, a foz do Rio Meriti enfrenta, rotineiramente, problemas associados ao assoreamento, dificultando a circulação e renovação das águas na baía, bem como sua navegabilidade. A foz do Saco de Engenho (Baía de Sepetiba, RJ), ao longo das últimas décadas, também tem recebido importantes contribuições de metais pesados (principalmente chumbo, cádmio, zinco e cobre) devido, dentre outros, ao passivo de uma antiga indústria metalúrgica localizada às adjacências do Saco de Engenho (Molisani et al., 2004; Monte, 2014). Neste sentido, vale ressaltar que o Porto de Sepetiba necessita frequentemente de intervenções de dragagem, para a atracagem adequada de navios em função da redução do calado operacional. No que se refere à bacia do Rio São Francisco, a mesma também recebe altas cargas de esgoto doméstico sem tratamento prévio adequado, além de seus tributários drenarem áreas intensamente urbanizadas e povoadas. Similarmente à foz do Rio Meriti, a foz do canal de São Francisco sofre com severos eventos de assoreamento (Molisani et al., 2004, Monte, 2017).

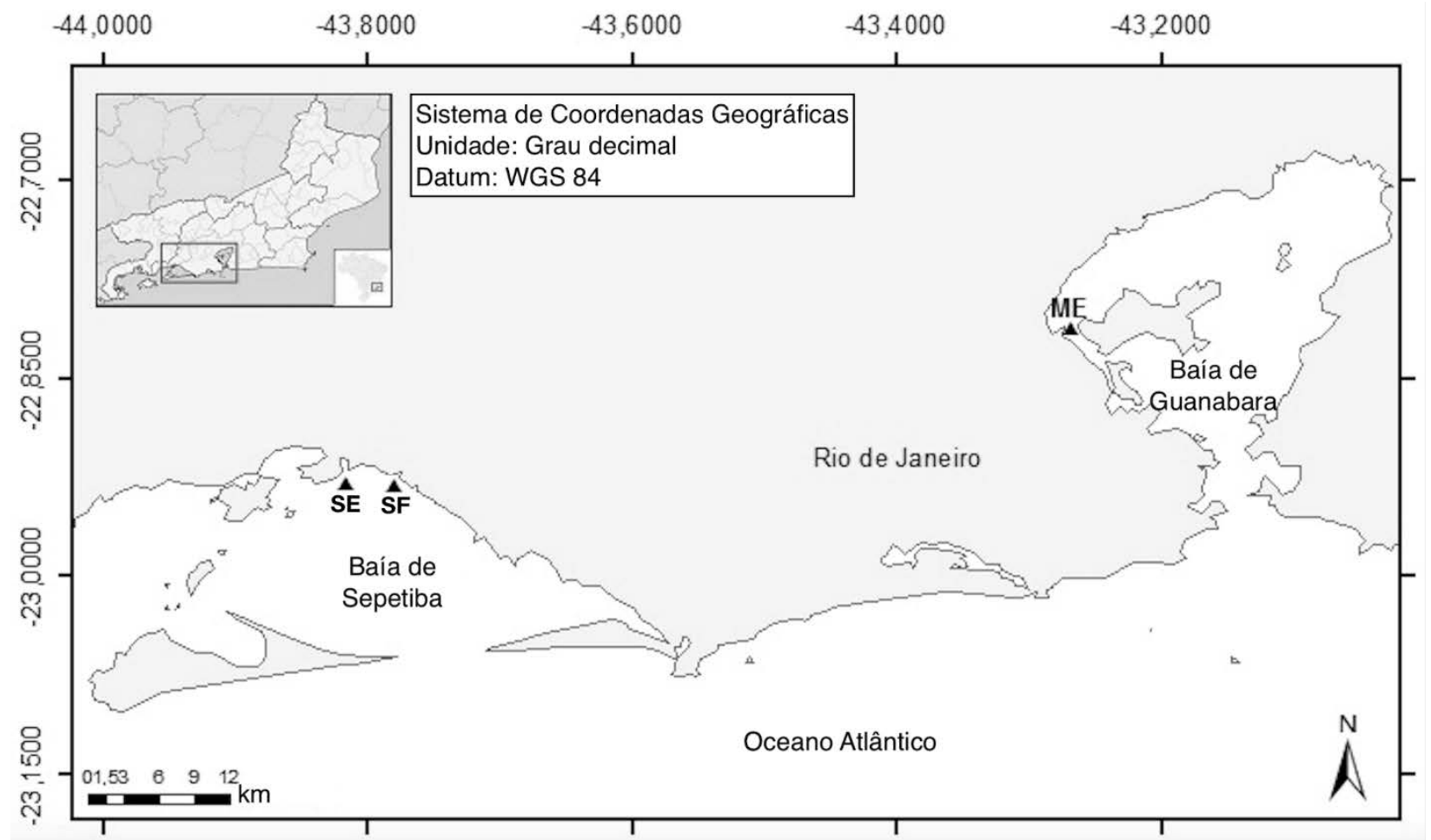

Figura 1 - Estações de coleta de sedimentos de dragagem nas Baías de Guanabara e de Sepetiba (RJ). SE = Saco do Engenho; $\mathrm{SF}=$ foz do canal de São Francisco; $\mathrm{ME}=$ foz do Rio Meriti.

\section{Amostragem de solo}

A fim de simular o descarte terrestre dos sedimentos, uma amostra de Latossolo foi coletada no município de Duque de Caxias (RJ) ( $22^{\circ} 41^{\prime} 34$. 2" S, 43 17 ' 14. 5”W). A escolha deste solo se deve à sua ampla distribuição geográfica no Brasil e ao seu vasto uso na agricultura brasileira. A amostra foi coletada com auxílio de trado manual e acondicionada em saco plástico. Em laboratório, o solo foi seco à temperatura ambiente e peneirado em $2,0 \mathrm{~mm}$.
A amostragem do solo foi realizada no Horizonte B.

A escolha do horizonte $\mathrm{B}$ se deve à simulação de um cenário de disposição no qual o sedimento possa ser usado como um regenerador de áreas degradadas como, por exemplo: (i) áreas de agricultura em que o horizonte A tenha sido erodido; (ii) áreas em que o horizonte A tenha sido removido por questões geotécnicas; e (iii) áreas em que o horizonte $\mathrm{A}$ tenha sido removido durante a lavra de minérios. Além disso, o horizonte B é o 
horizonte-diagnóstico do solo e, dessa forma, possibilita de forma muito mais efetiva a avaliação da influência das propriedades do solo na toxicidade de resíduos sólidos em ambientes terrestres.

Cesar et al. (2012) e Alamino et al. (2009) determinaram algumas propriedades físicas e químicas e mineralógicas de uma amostra de
Latossolo coletada no mesmo perfil de solo utilizado neste trabalho (Tabela 1). O Latossolo é de textura argilosa, ácido, de baixa fertilidade, possui altas concentrações de ferro e alumínio e baixo teor de matéria orgânica. Ademais, a mineralogia deste material é basicamente caulinítica (79\%), além da presença de goethita (8\%) e gibbisita (13\%).

Tabela 1 - Propriedades físicas e químicas e mineralógicas da amostra de Latossolo.

\begin{tabular}{|c|c|c|}
\hline \multicolumn{2}{|l|}{ Parâmetro } & \multirow{2}{*}{$\begin{array}{c}\text { Latossolo } \\
58 \\
\end{array}$} \\
\hline \multirow{3}{*}{$\begin{array}{c}\text { Textura } \\
(\%, n=3)\end{array}$} & Argila & \\
\hline & Silte & 6 \\
\hline & Areia & 36 \\
\hline \multirow{2}{*}{ pH $(n=3)$} & $\mathrm{H}_{2} \mathrm{O}$ & 4,2 \\
\hline & $\mathrm{KCl}$ & 3,6 \\
\hline \multirow{4}{*}{ Complexo sortivo $\left(\mathrm{cmol}_{\mathrm{c}} / \mathrm{dm}^{3}, \mathbf{n}=3\right)$} & $\mathrm{Mg}^{+2}+\mathrm{Ca}^{+2}$ & 0,2 \\
\hline & $\mathrm{K}^{+}$ & 0,02 \\
\hline & $\mathrm{Na}^{+}$ & 0,03 \\
\hline & CTC & 2,3 \\
\hline \multirow{2}{*}{ Concentrações totais $(\%, n=3)$} & $\mathrm{Al}$ & 26,4 \\
\hline & $\mathrm{Fe}$ & 11,3 \\
\hline Matéria Orgânica (\%, n = 3) & & 0,22 \\
\hline
\end{tabular}

Fonte: Adaptado de Cesar et al. (2012) e Alamino et al. (2009). CTC= capacidade de troca catiônica.

\section{Determinação do pH e da matéria orgânica}

A determinação do $\mathrm{pH}$ foi realizada por meio de eletrodo combinado imerso, na proporção de 1:2,5 (solo:água) (EMBRAPA, 1997), com o emprego de equipamento da marca DIGIMED. A quantificação do carbono orgânico (COT) foi efeituada em $1 \mathrm{~g}$ de amostra, previamente submetida à lavagem com ácido clorídrido (HCl), para remoção do carbono oriundo dos carbonatos (não-orgânicos). Após esta etapa, a amostra foi submetida à pirólise no Equipamento LECO SNS2000, sendo o teor de Carbono Orgânico Total (COT) determinado por análise elementar. A matéria orgânica foi obtida a partir da multiplicação do valor de COT por 1,724 (EMBRAPA, 1997).

\section{Determinação de metais pesados}

A determinação das concentrações totais de alumínio $(\mathrm{Al})$, cádmio $(\mathrm{Cd})$, cobre $(\mathrm{Cu})$, cromo $(\mathrm{Cr})$, ferro $(\mathrm{Fe})$, níquel $(\mathrm{Ni})$, chumbo $(\mathrm{Pb})$, zinco (Zn) e manganês (Mn) em amostras biológicas, de solo e sedimento foram determinadas via solubilização de $1 \mathrm{~g}$ de amostra em $40 \mathrm{ml}$ de uma mistura ácida de $\mathrm{HF}: \mathrm{HCl}: \mathrm{HClO}_{4}$ (2:1:1). A concentração de metais nos extratos foi quantificada por espectrometria de emissão atômica de plasma acoplada indutivamente (ICPOES; Horiba Jobin Yvon, Ultima 2). Os limites de detecção foram de: $0,4 \mathrm{mg} / \mathrm{kg}$ para Al; 0,1 mg/kg para Cd; 0,2 mg/kg para Cu; 0,01 mg/kg para Cr; 0,4 mg/kg para Fe; 0,4 mg/kg, para Mn; 0,2 mg/kg para Ni; 0,2 mg/kg para $\mathrm{Pb} ; 1,4 \mathrm{mg} / \mathrm{kg}$ para $\mathrm{Zn}$. As concentrações de metais nos sedimentos foram comparadas aos valores orientadores estabelecidos pela legislação brasileira para a disposição de sedimentos dragados em ambientes aquáticos (CONAMA 454/2012) e em solo (CONAMA 420/2009).

\section{Fatores de enriquecimento de metais}

O fator de enriquecimento é utilizado para avaliar o grau de contaminação associado ao input antropogênico de metais nos sedimentos (Ghrefat et., al. 2011; Karageorgis et. al., 2012). A identificação de possíveis anomalias antropogênicas nas concentrações de metais nos sedimentos é verificada através da normalização dos dados frente a um elemento de caráter conservativo e de origem predominantemente litogênica, como Al, Fe, ou Sc. Kemp et. al. (1976) introduziu uma equação (Equação I) designada como Fator de Enriquecimento (FE), utilizando o Al como elemento de referência para normalização (Solomons \& Förstner, 1984), empregada neste estudo:

$$
\mathrm{FE}=\frac{\left(\mathrm{M}_{\mathrm{i}} / \mathrm{Al}\right)_{\text {amostra }}}{\left(\mathrm{M}_{\mathrm{i}} / \mathrm{Al}\right)_{\text {background }}} \quad \text { (Equação I) }
$$

onde:

$\mathrm{Mi}=$ metal de interesse; $\mathrm{Al}$ = concentrações de 
Al no background no folhelho médio (Turekian \& Wedepohl, 1961) ou do background regional.

Valores o FE $>1$ indicam um enriquecimento em relação ao background, sugerindo a hipótese de que sua presença pode ser decorrente de atividades antropogênicas (Aquavita et. al. 2010). Em função da variabilidade natural, considera-se que o $\mathrm{FE}$ entre 0,5 e 2 como oriundos de contribuição natural, enquanto FE $>2$ indica a contribuição antrópica e/ou de processos biológicos.

Os valores de background foram obtidos a partir de trabalhos prévios que determinaram as concentrações de metais na base de testemunhos de sedimentos nas baías de Guanabara (Monteiro et al., 2012) e de Sepetiba (Gomes et al., 2009). Na ausência de valores na literatura, utilizaram-se as concentrações determinadas no folhelho médio (Turekian \& Wedepohl, 1961). A escolha pelo folhelho médio se deve ao fato de que se trata de uma rocha sedimentar de granulometria fina e alto teor de matéria orgânica, que, portanto, reúne atributos que favorecem a fixação de metais. Para a Baía de Guanabara, foram utilizados os seguintes valores (em mg/kg): $\mathrm{Cr}=90 ; \mathrm{Cu}=2,7 ; \mathrm{Ni}=8,3$; 14.9; $\mathrm{Zn}=70,2$; e Fe $=31607$. Para a Baía de Sepetiba, os valores de background foram (em $\mathrm{mg} / \mathrm{kg}): \mathrm{Cr}=9.3 ; \mathrm{Cu}=8 ; \mathrm{Ni}=8 ; \mathrm{Pb}=20 ; \mathrm{Zn}=54$; $\mathrm{Fe}=15900$ ).

\section{Bioensaios com minhocas (Eisenia andrei)}

O bioensaio agudo E. andrei foi baseado na American Society for Testing and Materials (ASTM (2004). Foram preparadas quatro (4) réplicas de $500 \mathrm{~g}$ de material por dosagem de sedimento no solo. As dosagens de sedimento dragado aplicadas aos solos foram baseadas em ensaios prévios realizados por Cesar et. al. (2017), que estudaram a toxicidade destas mesmas amostras de sedimento em solos artificiais. Sendo assim, as doses aplicadas foram: 6, 12, 18, 24 e $30 \%$, onde $0 \%$ = solo puro. Quando necessário, outras doses de sedimento foram aplicadas visando à obtenção de relações dose-resposta mais claras.

$\mathrm{O}$ teste foi realizado em recipientes plásticos cilíndricos (3,8 cm de diâmetro e 6,4 cm de altura). A umidade do solo foi previamente ajustada para a sua 40 - 60 \% da capacidade de retenção de água, conforme descrito em Natal-da-Luz et. al. (2009). Foram adicionados dez (10) organismos adultos com peso semelhante (variando de 300 a $600 \mathrm{mg}$ ) em cada réplica, de forma a obter populações mais homogêneas do ponto de vista etário e de biomassa. Antes de serem inseridos nos ensaios, os organismos foram deixados sobre papel absorvente umedecido com água destilada por $24 \mathrm{~h}$, para o esvaziamento do conteúdo intestinal, para eliminar eventuais interferências na determinação da biomassa corporal dos animais. Após 14 dias de exposição, sob temperatura controlada $\left(20 \pm 2{ }^{0} \mathrm{C}\right)$ e iluminação constante, contabilizou-se o número de organismos sobreviventes. A avaliação da variação da biomassa corporal dos animais é realizada através da diferença entre peso médio inicial e o peso médio final dos organismos inseridos na réplica, antes e após a exposição.

Réplicas adicionais com solo artificial tropical puro também foram preparadas para garantia dos resultados dos testes. Para validação do ensaio, são aceitos até $10 \%$ de mortalidade em solo artificial (ASTM, 2004). O solo artificial foi preparado de acordo com Garcia (2004), e é composto de 70\% de areia de quartzo, 20\% de caulim e 10\% de pó da fibra de casca de coco.

O número de minhocas sobreviventes nos testes de toxicidade aguda foi utilizado para estimar a dose de sedimento dragado capaz de causar a morte de $50 \%$ dos organismos expostos ( $\mathrm{CL}_{50}$ ), através da análise de PriProbit (Sakuma, 1998). Mudanças significativas na biomassa corporal dos organismos e de mortalidade encontrada nas misturas solo:sedimento foram compradas ao Latossolo puro (solos não contaminados - $0 \%$ ) através do teste de Kruskall-Wallis.

\section{RESULTADOS E DISCUSSÃO}

\section{Determinação do pH e da matéria orgânica}

Os resultados da determinação do $\mathrm{pH}$ e dos teores de matéria orgânica nos sedimentos puros estão demonstrados na tabela 2 . Os valores de $\mathrm{pH}$ indicam que o sedimento da foz do Rio Meriti é o mais ácido, um possível reflexo das severas condições de assoreamento, anoxia e eutrofização comumente apontadas para este ponto de coleta (Monte, 2014; Machado et al., 2014). O elevado teor de matéria orgânica obtido para este ponto (4,9\%) parece confirmar as condições redutoras (de anoxia), e é provavelmente decorrente, sobretudo, do despejo de esgoto doméstico sem pré-tratamento adequado e de baixos níveis de circulação de águas (Machado et al., 2014). Vale também ressaltar que valores mais ácidos de $\mathrm{pH}$ podem facilitar a lixiviação de metais, aumento sua mobilidade geoquímica e biodisponibilidade (Cesar et al., 2014). 
Os valores de $\mathrm{pH}$ para os demais pontos de coleta foram relativamente semelhantes entre em si e menos ácidos se comparados ao valor de 4,6 determinado para o Rio Meriti.

Estes valores mais básicos podem estar relacionados à abundância de minerais carbonáticos nestes materiais, que tipicamente ocorrem em ambientes estuarinos e marinhos. Além disso, as concentrações de matéria orgânica foram mais baixas para o Saco do Engenho e para o Sedimento do Canal São Francisco, o que pode sugerir a ocorrência de condições menos anóxicas e/ou contribuições comparativamente menos importantes de esgoto doméstico.

Em todo o caso, adição de sedimento dragado ao Latossolo (cujo pH já é ácido, 4,2) aparentemente não alterou significativamente o $\mathrm{pH}$ das misturas solo: sedimento com os sedimentos estudados, sendo que os valores variaram entre 4,5 e 5 para todas as misturas. Portanto, neste caso em específico, é provável que o pH tenha exercido papel de menor relevância na toxicidade.

Tabela 2 - Valores de pH e concentrações de matéria orgânica nos sedimentos dragados puros.

\begin{tabular}{c|c|c|c}
\hline Parâmetro & Saco do Engenho & Foz do Rio Meriti & Foz do C. São Francisco \\
\hline pH & 5,6 & 4,6 & 5,1 \\
\hline Matéria Orgânica (\%) & 1,8 & 4,9 & 0,93 \\
\hline
\end{tabular}

\section{Determinação de metais pesados}

As concentrações de metais nos sedimentos puros e sua comparação com os valores orientadores da legislação brasileira para disposição de sedimentos dragados em ambientes aquáticos e em solo estão demonstradas na tabela 3. Todos os sedimentos apresentaram a concentração de pelo menos um (1) metal excedendo os limites estipulados pela Resolução CONAMA 454/2012, indicando que os materiais são impróprios para disposição final em zonas marinhas (Tabela 3). Em termos de número de concentrações excedentes ao CONAMA 454, foi observada a seguinte ordem: Rio Meriti $=$ Saco de Engenho $>$ Foz do canal de São Francisco. Na foz do Rio Meriti, o único metal em conformidade com o CONAMA 454 foi o Cd, cuja concentração se encontra abaixo do limite de detecção. No Saco de Engenho, somente a concentração de $\mathrm{Cu}$ se encontra em concordância com o CONAMA 454. Por outro lado, na foz do Canal de São Francisco, com exceção do Zn, todos os metais analisados exibem concentrações abaixo daquelas preconizadas pelo CONAMA 454. Em termos de número absoluto de elementos metálicos acima do Nível 2 (alta probabilidade de efeitos tóxicos à biota aquática), vale destacar o Saco de Engenho (Cd, Ni e Zn), seguido do sedimento da foz do Rio Meriti (Zn). O sedimento da foz do Canal de São Francisco não apresentou nenhuma concentração acima do referido limite.

No âmbito das comparações das concentrações de metais com a Resolução
CONAMA 420/2009 para qualidade de solos, o único material considerado apto para disposição terrestre foi o sedimento da foz do Canal de São Francisco, tendo em vista que as suas concentrações se encontram abaixo dos valores orientadores. No caso do sedimento da foz do Rio Meriti, somente o teor de Cd se apresentou em concordância com a legislação supracitada. No Saco de Engenho, somente as concentrações de $\mathrm{Pb}$ e Cu estavam abaixo dos limites estabelecidos pelo CONAMA 420. O número de concentrações excedentes ao CONAMA 420 foi maior no Rio Meriti, seguido pelo Saco do Engenho. No sedimento da foz do Rio Meriti, o único metal que excedeu o limite de intervenção (para risco à saúde humana) foi o Zn, enquanto no Saco do Engenho, além do $\mathrm{Zn}$, o Cd também se encontrava acima do referido limite. Todas as demais concentrações excedentes mencionadas se referem a valores acima do limite de prevenção (para risco ecológico).

Os resultados referentes de FE de metais nos sedimentos puros estão indicados na Tabela 3 . Tendo em vista que valores acima de duas (2) unidades de FE apontam contribuição antrópica significativa de contaminação, na foz do Rio Meriti observa-se valores acima deste limite para $\mathrm{Cu}, \mathrm{Pb}, \mathrm{Ni}$ e $\mathrm{Zn}$. No Saco de Engenho, os FEs para o Cd, Cr, Zn e Ni também exibem valores acima de duas (2) unidades, enquanto no sedimento da foz do Canal de São Francisco somente o $\mathrm{Cr}$ excedeu o valor de 2. Os dois maiores valores de FE obtidos dentre todos os metais e sedimentos dragados estudados dizem 
respeito ao $\mathrm{Zn}$ e o $\mathrm{Cu}$, com valores de 10,7 e 33,3, Meriti, indicando contribuição antrópica extrerespectivamente, no sedimento da foz do Rio mamente intensa de contaminação.

Tabela 3 - Concentrações e FE de metais nos sedimentos dragados puros, incluindo comparação com os valores orientadores propostos para disposição de sedimentos dragados em ambientes aquáticos (CONAMA 420/2009) e em solo (CONAMA 454/2012). DI = dado indisponível (concentração abaixo do limite de detecção).

\begin{tabular}{|c|c|c|c|c|c|c|c|c|c|c|}
\hline \multirow{3}{*}{ Metal } & \multirow{2}{*}{\multicolumn{2}{|c|}{$\begin{array}{c}\text { Foz do Rio } \\
\text { Meriti }\end{array}$}} & \multirow{2}{*}{\multicolumn{2}{|c|}{$\begin{array}{c}\text { Saco do } \\
\text { Engenho }\end{array}$}} & \multirow{2}{*}{\multicolumn{2}{|c|}{$\begin{array}{l}\text { Foz do C. São } \\
\text { Francisco }\end{array}$}} & \multicolumn{2}{|c|}{ CONAMA 454} & \multicolumn{2}{|c|}{ CONAMA 420} \\
\hline & & & & & & & \multirow{2}{*}{$\frac{\text { Nível } 1}{\mathrm{mg} / \mathrm{kg}}$} & \multirow{2}{*}{$\frac{\text { Nível } 2}{\mathrm{mg} / \mathrm{kg}}$} & \multirow{2}{*}{$\begin{array}{c}\text { Prevenção } \\
\mathrm{mg} / \mathrm{kg}\end{array}$} & \multirow{2}{*}{$\frac{\text { Intervenção }}{\mathrm{mg} / \mathrm{kg}}$} \\
\hline & $\mathrm{mg} / \mathrm{kg}$ & $\mathrm{FE}$ & $\mathrm{mg} / \mathrm{kg}$ & $\mathrm{FE}$ & $\mathrm{mg} / \mathrm{kg}$ & $\mathrm{FE}$ & & & & \\
\hline Cd & $<0,10$ & DI & 7,2 & $6,9 *$ & $<0,10$ & DI & 1,2 & 7,2 & 1,3 & 3 \\
\hline $\mathrm{Cr}$ & 111 & 1,0 & 133 & $4,6^{*}$ & 43,5 & $2,2 *$ & 81 & 370 & 75 & 150 \\
\hline $\mathrm{Cu}$ & 111 & $33,3 *$ & 15 & 0,6 & 11,1 & 0,6 & 34 & 270 & 60 & 200 \\
\hline $\mathrm{Ni}$ & 42,9 & $4,2^{*}$ & 58,4 & $2,3^{*}$ & 13 & 0,7 & 20,9 & 51,6 & 30 & 70 \\
\hline $\mathbf{P b}$ & 90,9 & $4,9 *$ & 36,8 & 0,6 & 30,2 & 0,7 & 46,7 & 218 & 72 & 180 \\
\hline Zn & 926 & $10,7^{*}$ & 1200 & $7,2^{*}$ & 206 & 1,8 & 150 & 410 & 300 & 450 \\
\hline
\end{tabular}

Notas: Nível 1 = limiar de baixa probabilidade de efeitos tóxicos à biota aquática. Nível 2 = limiar de alta probabilidade de efeitos tóxicos à biota aquática. Prevenção = limite para ocorrência de efeitos adversos a receptores ecológicos do solo. Intervenção = limite de risco à saúde humana. $*$ = valores de FE que excedem duas (2) unidades e que indicam fonte antrópica de contaminação.

A tabela 4 apresenta uma comparação das concentrações de metais obtidas nos sedimentos estudados com materiais dragados de outras localidades do Estado do Rio de Janeiro. Vale destacar que o método analítico empregado para a determinação de metais nos materiais indicados na Tabela 5 é a mesmo utilizado para a quantificação destes elementos neste trabalho. Em comparação a outros sedimentos, as concentrações encontradas no Saco do Engenho, seguido do sedimento da Foz do Rio Meriti,

foram as maiores. A concentração de $\mathrm{Zn}$ na foz do Saco de Engenho, por exemplo, chega a exceder quase três (3) vezes e onze (11) vezes os teores encontrados no sedimento dragado do Porto do Rio de Janeiro e nos sedimentos dragados dos Rios Botas e Sarapuí na baixada fluminense, respectivamente. No caso da Foz do Rio Meriti, vale ainda ressaltar que a concentração de $\mathrm{Pb}$ é somente excedida pelo teor encontrado no sedimento dragado do Canal do Cunha.

Tabela 4 - Concentrações de metais tóxicos (em mg/kg) obtidas neste trabalho e comparação com valores determinados em sedimentos dragados de outras localidades do Estado do Rio de Janeiro.

\begin{tabular}{|c|c|c|c|c|c|c|c|}
\hline Área de Estudo & Cd & $\mathrm{Cr}$ & $\mathbf{C u}$ & $\mathbf{N i}$ & $\mathbf{P b}$ & Zn & Referência \\
\hline Saco do Engenho (Baía de Sepetiba, RJ) & 7,2 & 133 & 15 & 58 & 37 & 1200 & \multirow{3}{*}{ Este estudo } \\
\hline Foz do rio Meriti (Baía de Guanabara, $\mathrm{RJ}$ ) & $<0,10$ & 111 & 111 & 43 & 91 & 926 & \\
\hline $\begin{array}{c}\text { Foz do canal de São Francisco (Baía de } \\
\text { Sepetiba) }\end{array}$ & $<0,10$ & 44 & 11 & 13 & 30 & 206 & \\
\hline $\begin{array}{l}\text { Lagoa Rodrigo de Freitas (Rio de Janeiro, } \\
\text { RJ) }\end{array}$ & $<0,10$ & 42 & 52 & 16 & 74 & 233 & Vezzone et al. (2018) \\
\hline Canal do Cunha (Baía de Guanabara, RJ) & - & 95 & 92 & 20 & $\begin{array}{c}12 \\
4\end{array}$ & 329 & Cesar et. al. (2014a) \\
\hline Rios Botas e Sarapuí (Baixada Fluminense) & $<0,06$ & 94 & 26 & 8,6 & 19 & 114 & Cesar et. al. (2014b) \\
\hline Porto do Rio de Janeiro (RJ) & $<0,10$ & 104 & 114 & 48 & 87 & 453 & Monte et. al. (2017) \\
\hline
\end{tabular}

Tendo em vista os valores de FE obtidos e a análise comparativa com sedimentos dragados de outras localidades do Estado do RJ, na foz do Saco de Engenho as concentrações elevadas de $\mathrm{Cd}, \mathrm{Zn}$ e Ni estão possivelmente relacionadas à lixiviação e/ou erosão de antigos rejeitos de uma

antiga instalação metalúrgica atualmente desativada. Assim, vale destacar também o Saco do Engenho foi a única área de estudo a apresentar concentração de Cd acima do limite de detecção e, além disso, os maiores valores de FE obtidos nesta área foram para Cd e Zn. A foz do 
Saco de Engenho necessita de dragagem frequente devido ao Porto de Sepetiba e, neste sentido, atenção especial deve ser dada aos referidos metais.

\section{Bioensaios com minhocas (Eisenia andrei)}

Os dados referentes à sobrevivência das minhocas expostas a diferentes doses de sedimento dragado da Foz do Saco de Engenho, do Rio Meriti e do Canal de São Francisco estão demonstrados na figura 2. Na foz do Saco de Engenho, os resultados indicam altos níveis de sobrevivência até a dose de 12 \% (acima de 95\%), seguida de uma queda abrupta e significativa na dosagem de $14 \%$ ( $p<0,05$, Kruskall-Wallis). A CL50 (expressa em \% de sedimento adicionado ao solo) determinada para esta área de estudo foi de 13,06 \%. Para o sedimento da foz do Rio Meriti, constatou-se queda brusca e significativa da sobrevivência a partir da dose de $12 \%$ ( $<<0,05$, KruskallWallis). Neste caso, de $12 \%$ para $16 \%$, observase redução de $60 \%$ da sobrevivência. A partir da dose de $20 \%$, todos os organismos expostos morreram.

A $\mathrm{CL}_{50}$ estimada para esta área de estudo foi de 14,60 \%. Por fim, no Canal de São Francisco, até a dosagem de $24 \%$, não houve morte significativa de organismos. Uma redução significativa da sobrevivência foi somente detectada para a dose de $32 \%$ (p $<0,05$, Kruskall-Wallis), com CL50 estimada em 28,02 \%. Não houve variação significativa de biomassa corporal dos animais para nenhum dos sedimentos.

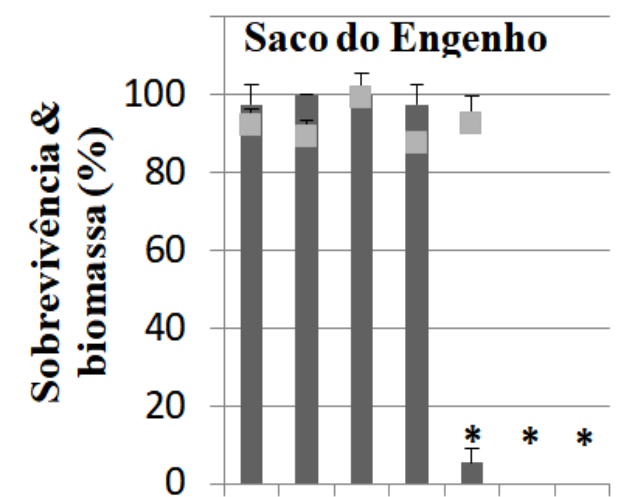

0. 4. 8. 12.14.16.20.

\section{Rio Meriti}

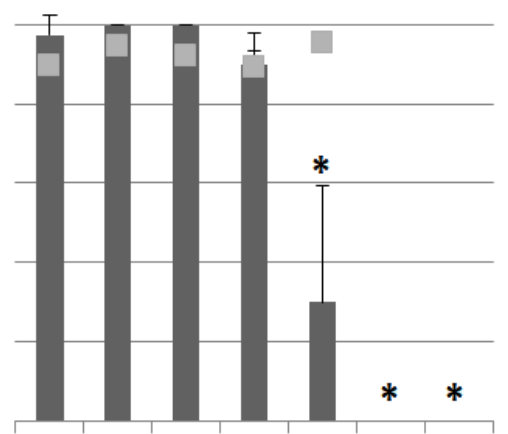

0. 4. 8. 12. 16. 20. 24 .

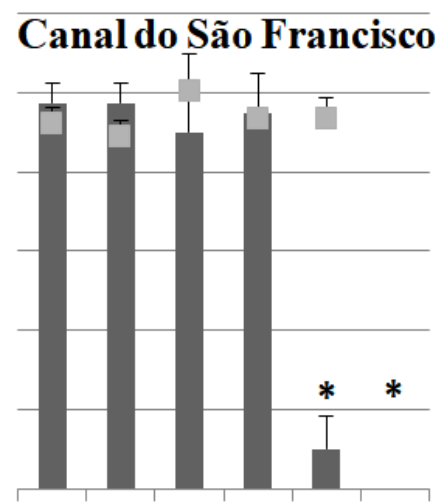

0. 8. 16. 24. 32. 40 .

\section{Doses de sedimento dragado $(\%)$}

\section{- Sobrevivência Biomassa}

Figura 2 - Sobrevivência e variações da biomassa de minhocas (Eisenia andrei) após exposição a Latossolo tratado com diferentes doses de sedimentos dragados da foz do Saco de Engenho (Baía de Sepetiba, RJ), Rio Meriti (Baía de Guanabara, RJ) e Canal de São Francisco (Baía de Sepetiba, RJ). * = redução significativa da sobrevivência em relação ao controle $(0 \%)$ (Kruskall Wallis, p < 0,05).

A tabela 5 apresenta uma comparação entre os valores de $\mathrm{CL}_{50}$ obtidas neste estudo com aquelas determinadas por Cesar et al. (2017), que estudaram a toxicidade destas mesmas amostras de sedimentos em solos artificiais, com o emprego de bioensaio agudo com $E$. andrei. Para todos os sedimentos estudados, a $\mathrm{CL}_{50}$ obtida em Latossolo neste trabalho foi menor do que aquela determinada por Cesar et al. (2017) em solos artificiais (Tabela 6), sugerindo que a toxicidade é maior em Latossolo. Esta constatação pode estar atrelada (i) ao fato de que o Latossolo é um solo ácido ( $\mathrm{pH}=4,2$, enquanto o solo artificial tem $\mathrm{pH}$ em torno de 5,5 - 6,5) e, portanto, tende a lixiviar mais facilmente metais pesados e sais, tornandoos potencialmente mais biodisponíveis para absorção via epiderme das minhocas.

Neste sentido, vale ressaltar, no caso das minhocas, que a absorção de metais via epiderme é mais importante do que a via de ingestão de solo. Vijver et al. (2003), ao propor um método para distinguir a absorção dermal e via ingestão com base no selamento da cavidade oral das minhocas (Eisenia fetida), indicaram que a assimilação de metais pelos organismos é predominantemente via exposição dérmica; e (ii) ao fato de que os teores de nutrientes (fertilidade natural) e de matéria orgânica em Latossolo são muito baixos se comparados ao solo artificial, que conta com $10 \%$ do pó da casca de coco. Neste contexto, a disponibilidade de alimento para minhocas em Latossolo é baixa, independente da adição de sedimento. 
Assim, é válido supor que organismos mal alimentados tenderiam a ser menos resistentes (sistema imunológico mais débil) quando expostos a agentes tóxicos no solo (Cesar et al., 2012).

A tabela 6 apresenta uma comparação entre os valores de $\mathrm{CL}_{50}$ determinados neste trabalho e dados de $\mathrm{CL}_{50}$ obtidos para sedimentos dragados oriundos de outros ecossistemas aquáticos do Estado do Rio de Janeiro, em misturas com a mesma amostra de Latossolo utilizada neste presente trabalho e com o emprego de bioensaio agudo com E. andrei. Estes dados apontam que, embora as concentrações de metais nos sedimentos do Saco de Engenho e da foz do Rio Meriti sejam relativamente mais altas do que as demais áreas (Tabela 6), os valores de $\mathrm{CL}_{50}$ para estas referidas áreas são comparativamente maiores, sugerindo que a toxicidade é menor em relação a outros sistemas hídricos do Estado do Rio de Janeiro. Esta observação pode estar relacionada à (i) presença de sais marinhos nestes materiais, com potencial de causar efeitos tóxicos à biota terrestre (Owojori et al. 2009). Em trabalhos futuros, a toxicidade de sais para minhocas deverá ser estudada em mais detalhe em solos tropicais; e (ii) presença de outros agentes tóxicos não avaliados neste trabalho, tais como hidrocarbonetos de petróleo, fármacos, hormônios e agrotóxicos.

Tabela 5 - Comparação entre os valores de dose letal de sedimento dragado a 50 \% dos organismos (CL50) obtidos para os sedimentos estudados em misturas com Latossolo e solo artificial.

\begin{tabular}{c|c|c}
\hline \multirow{2}{*}{ Área de Estudo } & CL50 em Latossolo (\%) & CL50 em Solo Artificial (\%) \\
\cline { 2 - 3 } & (Este estudo) & (Cesar et al., 2017) \\
\hline Foz do Saco do Engenho & 13,06 & 16,26 \\
\hline Foz do Rio Meriti & 14,6 & 17,43 \\
\hline Foz do Canal de São Francisco & 28,02 & DI* \\
\hline
\end{tabular}

Nota: DI = dado indisponível (neste caso, a mais alta dosagem aplicada, de 30\%, não causou morte significativa às minhocas).

Tabela 6 - Valores de dose letal de sedimento dragado a $50 \%$ dos organismos (CL50) e comparação com valores determinados para outras localidades do Rio de Janeiro. DI = dado indisponível. * = o sedimento puro (100\%) não causou morte significativa aos organismos.

\begin{tabular}{c|c|c}
\hline Área de Estudo & CL50 (\%) & \multirow{2}{*}{ Referência } \\
\cline { 1 - 2 } Saco do Engenho (Baía de Sepetiba, RJ) & 13,06 & \multirow{2}{*}{ Este estudo } \\
\cline { 1 - 2 } Foz do rio Meriti (Baía de Guanabara, RJ) & 14,6 & \\
\cline { 1 - 2 } Foz do canal de São Francisco (Baía de Sepetiba) & 28,02 & \multirow{2}{*}{ Vezzone et al. (2018) } \\
\hline Lagoa Rodrigo de Freitas (Rio de Janeiro, RJ) & 9,2 & Cesar et. al. (2014a) \\
\hline Canal do Cunha (Baía de Guanabara, RJ) & 9,9 & Cesar et. al. (2014b) \\
\hline Rios Botas e Sarapuí (Baixada Fluminense) & DI* & Monte et. al. (2017) \\
\hline Porto do Rio de Janeiro (RJ) & 7,71 &
\end{tabular}

Nota: DI = dado indisponível. * = o sedimento puro (100\%) não causou morte significativa aos organismos.

\section{CONCLUSÕES}

Os sedimentos avaliados foram tóxicos às minhocas em cenário de disposição em Latossolo, sendo o sedimento dragado do Saco do Engenho $($ CL50 = 13,06\%) o de maior toxicidade, seguido da foz do Rio Meriti (CL50 $=14,60 \%)$ e do Canal de São Francisco (28,02\%). Neste sentido, o bioensaio agudo com minhocas parece ter sido adequado para uma avaliação preliminar da toxicidade destes sedimentos em cenário de disposição terrestre. Além disso, a aplicação deste bioensaio possibilitou avaliar a influência potencial do substrato pedológico na toxicidade, tendo em vista que os valores de CL50 obtidos em solos artificiais (Cesar et al., 2017) foram maiores do que aqueles em Latossolo. Tal fato aponta para a necessidade de se revisar os valores orientadores da legislação brasileira com base na variação das propriedades das principais classes pedológicas brasileiras, de modo a adequar tais valores às particularidades da região tropical. Além disso, recomenda-se a realização de bioensaios com organismos de outros níveis taxonômicos e tróficos do solo (por exemplo, colêmbolos, enquitreídos e vegetais) de forma a prognosticar, de forma mais consistente, o risco ecológico associado à disposição terrestre de materiais dragados. 


\section{AGRADECIMENTOS}

Os autores gostariam de agradecer ao apoio financeiro do CNPq, através de concessão de bolsa a Danielle Siqueira (IC - CNPq), de fundamental importância à realização deste trabalho.

\section{REFERENCIAS}

AQUAVITA, A.; PREDONZANI, S.; MATTASSI, G.; ROSSIN, P.; TAMBERLICH, F.; FALOMO, J.; VALIC, I. Heavy metal contents and distribution in coastal sediments of the Gulf of Trieste (Northern Adriatic Sea, Italy). Water, Air, \& Soil Pollution, v. 211, n. 1, p. 95-111, 2010.

ALAMINO, R.C.J.; POLIVANOV H.; CAMPOS, T.M.P.C.; SILVA V.H.G.; SANTOS L.V.; MENDES J.C. Biodisponibilidade de cádmio em latossolo acrescido de lodo de esgoto. Anuário do Instituto de Geociências, v. 30, n. 2, p. 45-54, 2007.

ALMEIDA, M.S.S.; BORMA, L.S.; BARBOSA, M.C. Land disposal of river and lagoon dredged sediments. Engineering Geology, v. 60, n. 1-4), p. 21-30, 2001.

ALVES, P.R.L.; NATAL-DA-LUZ, T.; SOUSA, J.P.; CARDOSO, E.J.B.N. Ecotoxicological characterization of sugarcane vinasses when applied to tropical soils. Science of the Total Environment, v. 526, p. 222-232, 2015.

ASTM - American Society for Testing and Materials. Standard Guide For Conducting Laboratory Soil Toxicity Or Bioaccumulation tests with the Lumbricid Earthworm Eisenia fetida and the enchytraeid potworm Enchytraeus albidus, 2004.

BIANCHI, M.O. Ensaios Ecotoxicológicos como ferramenta para avaliação do impacto ambiental de resíduos de mineração sobre o solo. Rio Janeiro, 2013. Tese (Doutorado Ciência do Solo - Agronomia), Universidade Federal Rural do Rio de Janeiro.

CESAR, R.G.; SILVA, M.B.; COLONESE, J.P.; BIDONE, E.D.; EGLER, S.; CASTILHOS, Z.C.; POLIVANOV, H. Influence of the properties of tropical soils in the toxicity and bioavailability of heavy metals in sewage sludge-amended lands. Environmetal Earth Sciences, v. 66, p. 2281-2292, 2012.

CESAR, R.G.; RODRIGUES, A.P.; SANTOS, M.C.; SENDEROWITZ, S.; COLONESE, J.; MOREIRA, C.; BERTOLINO, L.C.; EGLER, S.G.; CASTILHOS, Z.C.; MADDOCK, J. Ecotoxicidade e biodisponibilidade de metais em solos impactados por rejeitos industriais em Queimados, RJ, Brasil. São Paulo. UNESP Revista Geociências, v. 32, p. 600610, 2014a.

CESAR, R.G.; NATAL-DA-LUZ, T.; SOUSA, J.P.; BIDONE, E.D., COLONESE, J.P.; CASTILHOS, Z.C.; EGLER, S.G.; POLIVANOV, $\mathrm{H}$. Disposal of dredged sediments in tropical soils: ecotoxicological effects on earthworms. Environmetal Monitoring and Assessment, v. 186, p. 1487-1497, 2014b.

CESAR, R.G.; RODRIGUES, A.P.; ROCHA, B.C.R.C.; CAMPOS, T.M.P.; MONTE, C.; DEALTRY, S.; CASTILHOS, Z.C.; MACHADO, W. Ecotoxicological assessment of dredged sediments from Guanabara and Sepetiba bays (Rio de Janeiro State, Brazil) using bioassays with earthworms. In: Araujo C, Shinn C (eds) Ecotoxicology in Latin America, Nova Publishers, New York, p. 309-324, 2017.

CORDEIRO, R.C.; SANTELLI, R.E.; MACHADO, W.; MOREIRA, L.S.; FREIRE, A.S.; BRAZ, B.F.; RIZZINIANSARI, N.; BIDONE, E.D.; MENICONI, M.F.G. Biogeochemical factors controlling arsenic distribution in a densely populated tropical estuary (Guanabara Bay, RJ, Brazil). Environmental Earth Sciences, v. 76, p. 1, 2017.

EMBRAPA-Empresa Brasileira de Pesquisa Agropecuária. Centro Nacional de Pesquisa de Solos. In: Manual de Métodos de Análises de Solo. Rio de Janeiro, RJ, p. 212.

GARCIA, M. Effects of pesticides on soil fauna: Development of ecotoxicology test methods for tropical regions. In: P.L.G. VLEK, M. DENICH, C. MARTIUS, N. GIESEN (Eds), Ecology and development series. V. 19, Cuvillier Verlag
Gottingen, 282 p, 2004.

GHREFAT, H; ABU-RUKAH, Y.; ROSEN, M. Application of geoaccumulation index and enrichment factor for assessing metal contamination in the sediments of Kafrain Dam, Jordan. Environmental Monitoring and Assessment, v. 178, n 1, p. 95-109, 2011.

GOMES, F.C.; GODOY, J.M.; GODOY, M.L.D.P.; CARVALHO, Z.L.; LOPES, T.R.; SANCHEZ-CABEZA, J.A.; LARCERDA, L.D.; WASSERMANN, J.C. Metal concentrations, fluxes, inventories and chronologies in sediments from Sepetiba and Ribeira Bays: A comparative study. Marine Pollution Bulletin, v. 59, p. 123 -133, 2009.

HINTO, J. \& VEIGA M.M. Earthworms as bioindicators of mercury pollution from mining and other industrial activities. Geochemical Exploration and Environment Analysis, v. 2, n. 3, p. 269-274, 2002.

KARAGEORGIS, A.; SIOULAS, A.; KRASAKOPOULOU, E.; ANAGNOSTOU, C.; HATIRIS, G.; KYRIAKIDOU, H.; VASILOPOULOS, K. Geochesmistry of surface sediments and heavy metal contamination assessment: Massolonghi lagoon complex, Greece. Environmental Earth Sciences, v. 65, n. 6, p. 1619-1629, 2012.

KEMP, A.L.W. \& THOMAS, R.L. Impact of man's activities on the chemical composition in the sediments of Lakes Ontario, Erie and Huron. Water, Air \& Soil Pollution, v. 5, p. 469-490, 1976.

MACHADO, W.; SANTELLI, R.E.; LOUREIRO, D.D.; OLIVEIRA, E.P.; BORGES, A.C.; LACERDA, L.D. Mercury accumulation in sediments along an eutrophication gradient in Guanabara Bay, southeast Brazil. Journal of the Brazilian Chemical Society, v. 19, n. 3, p. 569-575, 2008.

MACHADO, W.; BORRELLI, N.L.; FERREIRA, T.O.; MARQUES, A.G.B.; OSTERRIETH, M.; GUIZAN, C. Trace metal pyritization variability in response to mangrove soil aerobic and anaerobic oxidation processes. Marine Pollution Bulletin, v. 79, p. 365-370, 2014.

MOLISANI, M.M.; MARINS, R.V.; PARAQUETTI, H.H.M.; BIDONE, E.D.; LACERDA, L.D. Environmental changes in Sepetiba Bay, SE Brazil. Regional Environmental Change, v. 4, n. 1, p. 17-27, 2004.

MONTE, C. Análise da biodisponibilidade de metais a partir da ressuspensão de sedimentos no Saco do Engenho, Sepetiba. Rio de Janeiro. 2014. Dissertação (Mestrado Geociências-Geoquímica), Universidade Federal Fluminense.

MONTE, C. Comportamento de contaminantes metálicos em eventos de resuspensão de sedimentos portuários e estuarinos tropicais. Rio de Janeiro. 2017. Tese (Doutorado Geociências - Geoquímica), Universidade Federal Fluminense. MONTE, C.N.; RODRIGUES, A.P.C.; FREIRE, A.S.; SANTELLI, R.E.; MACHADO, W. Metal Bioavailability in Contaminated Estuarine Sediments from a Highly-Impacted Tropical Bay. Revista Virtual de Química, n. 9, v. 4, p. 20072016, 2017.

MONTEIRO, F.F.; CORDEIRO, R.C.; SANTELLI, R.E.; MACHADO, W.; EVANGELISTA, H.; VILLAR, L.S.; VIANA, L.C.A.; BIDONE, E.D. Sedimentary geochemical record of historical anthropogenic activities affecting Guanabara Bay (Brazil) environmental quality. Environmental Earth Sciences, v. 65, p. 1661-1669, 2012.

MUNNS, W.R.; BERRY, W.J.; DEWITT, W.T. Toxicity testing, risk assessment, and options for dredged material management. Marine Pollution Bulletin, v. 44, p. 294-302, 2002.

NASCIMENTO, J.R.; SABADINI-SANTOS, E.; CARVALHO, 
C.; KEUNECKE, K.A.; CESAR, R.; BIDONE, E.D. Bioaccumulation of heavy metals by shrimp (Litopenaeus schmitti): A dose-response approach for coastal resources management. Marine Pollution Bulletin, v. 114, p. 10011007, 2016.

NEUHAUSER, E.F.; LOEHR, R.C.; MILLIGAN, D.L.; MALECKI, M.R. Toxicity of metals to the earthworms Eisenia foetida. Biology and Fertilityof Soils, v. 1, p. 149-152, 1985. RODRIGUES, S.K.; ABESSA, D.M.S.; RODRIGUES, A.P.C.; SOARES-GOMES, A.; FREITAS, C.B.; SANTELLI, R.E.; FREIRE, A.S.; MACHADO, W. Sediment quality in a metalcontaminated tropical bay assessed with a multiple lines of evidence approach. Environmental Pollution, v. 228, p. 265276, 2017.

SAKUMA, M. Probit analysis of preference data. Applied Entomology Zoology, v. 33, p. 339-347, 1998.

SOLOMONS, W. \& FÖRSTNER, U. Metals in the Hydrocycle. Berlin: Springer. 1984.

TUREKIAN, K.K. \& WEDEPOHL, K.H. Distribution of elements in some major units of the earth’s crust. Geological Society of America Bulletin, v. 72, n. 2, p. 175-192, 1961.
VAN GESTEL, C.A.M; BORGMAN, E.E.F., VERWEIJ, R.A.; ORTIZ, M.D. The influence of soil properties on the toxicity of molybdenum to three species of soil invertebrates. Ecotoxicology and Environmental Safety, v. 74, p. 1-9, 2011. VEZZONE, M.; CESAR, R.; POLIVANOV, H.; SERRANO, A.; SIQUEIRA, D.; ABREU, L.; BIANCHI, M.; CORREIA, M.E.; CASTILHOS, Z.; DE CAMPOS, T. Ecotoxicological evaluation of dredged sediments from Rodrigo de Freitas Lagoon (Rio de Janeiro State, Brazil) using bioassays with earthworms and collembolans. Environmental Earth Sciences, v. 77, p. 743, 2018.

Submetido em 8 de março de 2019 Aceito para publicação em 3 de novembro de 2020 